\title{
PERFORMANCE ANALYSIS OF RANDOMIZED REVERSE AD HOC ON DEMAND DISTANCE VECTOR ROUTING PROTOCOL IN MANET
}

\author{
${ }^{1}$ Santhi, K. and ${ }^{2}$ B. Parvathavarthini \\ ${ }^{1}$ Department of Computer Applications, Anand Institute of Higher Technology, \\ OMR Road, Kazhipattur, Chennai, Tamilnadu, India \\ ${ }^{2}$ Department of Computer Applications, \\ St.Joseph's College of Engineering, OMR Road, Jeppiaar Nagar, Chennai, Tamilnadu, India
}

Received 2014-01-31; Revised 2014-02-11; Accepted 2014-04-30

\begin{abstract}
In high mobility, routing in Mobile Ad-Hoc Network is a very difficult task. In AODV and RAODV, all the data packets are travel through the same shortest path so the intruders can easily trace out the data path. The main objective of Randomized RAODV provides the multipath and then the paths are selected randomly for security purposes. Using randomized routing algorithm to choose the path randomly and then the data packets are travel through different path to reach the destination, so the hackers cannot know about what are the ways the data packets traverse. The performance of proposed RRAODV is compared with the existing routing protocol like AODV, RAODV in mobile network environment. Performance metrics such as packet delivery ratio, end to end delay and control packet overhead are evaluated using NS-2 based on the number of nodes and speeds. Simulation results shows RRAODV gives better performance than the existing protocols like AODV and RAODV for the above metrics. RRAODV is helpful to increase the performance of data transmission and security of data.
\end{abstract}

Keywords: Multipath, Ad Hoc On Demand Distance Vector (AODV), Security, Reverse Ad Hoc On Demand Distance Vector (RAODV), Security, Randomized Reverse Ad Hoc On Demand Distance Vector (RRAODV)

\section{INTRODUCTION}

A mobile Ad-hoc Network is a collection of interrelated nodes with no infrastructure and a multi hop wireless network with no centralized administration. The nodes in a mobile Ad-hoc network change dynamically such as some nodes join in a network, disconnect the network and also move at any time on the network. In the above reasons, the routing problem in MANET is more difficult than compared to the wired network.

Routing protocols in Ad-hoc network is grouped under three categories like proactive, reactive and hybrid routing protocols. In proactive type of routing protocols, the routing information in each node of a network is updated periodically whether the routing path is needed or not. DSDV and OLSR are example of proactive routing protocol.

Examples of Reactive routing protocols are AODV, DSR and TORA. This type of routing protocol maintain route only for data communication. These type of protocol helps to reduce routing overhead. Zone based Routing Protocol (ZRP) is an example of hybrid routing protocol. It combines the features of proactive and reactive routing protocols.

The rest of the paper is organized as follows. In the next section describes related work, third section describes proposed work, fourth section explains the Kazhipattur, Chennai, Tamilnadu, India 
simulation results and parameters. Finally, conclusion is added in the last section.

\section{RELATED WORK}

Split multipath routing (Lee and Gerla, 2001) is used to construct maximally disjoint paths in ad hoc networks. The destination node receives multiple RREQ packets and then selects the two maximum disjoint paths. AOMDV (Marina and Das, 2001) computes multiple loop-free and link-disjoint paths in AODV for route discovery but it cannot maintain the alternate path properly.

NMN-AODV protocol (Zangeneh and Mohammadi, 2011) based on AODV protocol and it improves the packet delivery ratio, reduces end-to-end delay and also uses two node disjoint routes between source and destination pair. MMDV (Mtibaa and Kamoun, 2006) protocol provides multipath and MPR based flooding in AODV and this protocol establishes multiple and disjoint path in a single route discovery process but cannot easy to handle congestion and collisions in high node density.

NDMR (Li and Cuthbert, 2004) protocol to reduce routing overhead and also achieves multiple nodedisjoint routing paths in AODV but it decrease the performance when increase the routing load. MPAOMDV (Sambasivam et al., 2004) protocol form the multiple path and it validate all the alternate path periodically using periodic update packets.

Kuo et al. (2009) author describes dynamic routing in DSDV for security purposes. The path between multiple sources to their multiple destinations is stored on a routing table and the paths are selected randomly by the source node and then the data packets are sent through this path.

RAODV (Kim et al., 2006) is the extension of AODV protocol. This protocol is helpful to decrease the loss of RREP packet and also prevent a retransmission of RREQ by the source node when the link is disconnected, so the congestion is reduced in a network.

Jaisankar and Saravanan (2010), authors compute multiple routes in a single route discovery and it produce node disjoint path and fail safe path for multiple routes. Path Hopping Based on Reverse AODV for security (Talipov et al., 2006) is the extension of RAODV. In this protocol, the source node stores multiple paths to the destination node and then the paths are selected sequentially from the list. SecMR (Kotzanikolaou et al., 2005) protocol together with AODV for security purposes and it discovers the complete set of non-cyclic and node-disjoint path. Taleb and Behzad (2012), the author performs the simulation study to compare the number of hops in selected path along route reply of AODV and RAODV. They conclude that RAODV data packets meet fewer hops in chosen path and remind energy is higher than AODV.

Khelifa and Maaza (2010), author computes the residual energy of nodes in RAODV, EA-RAODV (Gouda and Behera, 2012) is the extension of RAODV which is based on the combination of least hops, power and minimum remaining energy. RAODV (Das, 2013) has better performance than AODV for larger network size and low density networks with lower network mobility. Zarei et al. (2008) develop an algorithm and it is based on link/route stability estimation for decrease overhead of discovery and maintenance of routing. Humaira et al. (2011), Authors compare the performance of AODV and RAODV using the parameters like Throughput, Delay and packet delivery ratio and conclude that RAODV has better performance than AODV. An enhanced DOA (Vanitha and Parvathavarthini, 2013) to reduce the problem of position estimation error along with DTS and to estimate the nearest neighbor selection.

\section{PROPOSED WORK}

This protocol is the extension of Reverse-AODV protocol and it is based on distributed routing information. This one is helpful to improve the security of data transmission. This protocol has multipath routing path to the destination node. Multipath is more advantage in large networks and also provides load balancing. It is helpful to establish more than one path between source and destination node. If the link is failed in a network then the data packets are correctly and securely reach to the destination node using alternate paths to the destination node.

\subsection{Route Discovery}

Source node broadcast the RREQ packet to their neighborhood nodes within their transmission range. The content of the RREQ packet is as follows.

Broadcast ID is uniquely identifying the Route Request (RREQ) packet. The neighborhood node receives RREQ packet which is a destination node it prepares Reverse Route Request (R-RREQ) packet otherwise it forwards to the next neighborhood nodes and this process continues finally this packet reach to the destination nod. When an intermediate node receives multiple copies of the same RREQ packet then it accepts the first RREQ packet and drops the remaining RREQ packet (Fig. 1). 
The destination node only generates the R-RREQ packet and it makes this packet only when it receives the first RREQ packet from the source node. The format of the R-RREQ packet is as follows (Fig. 2).

Destination node broadcast the R-RREQ packet within the transmission range. When an intermediate node receives R-RREQ packet, if it already have the same R-RREQ packet then it drops it otherwise it forward to the next neighborhood nodes on the network. Each node maintains the routing table. The content of the routing table is as follows.

Source node collects the R-RREQ packet from the various neighborhood nodes. Based on the information of routing Table 1, source node selects the path randomly from the routing table for security purposes. Applying randomized algorithm to choose the path randomly on the routing table. Source node selects the path randomly on the routing table and it is used to forward the data packets.

\subsection{Route Maintenance}

Hello packet is used to detect whether the link is failed or not. When the link is failed in a network, neighborhood node of the failed link sends RERR packet to the source node.

\begin{tabular}{|c|c|c|}
\hline Type & Reserved & Hop Count \\
\hline \multicolumn{3}{|c|}{ Broadcast ID } \\
\hline \multicolumn{3}{|c|}{ Destination IP address } \\
\hline \multicolumn{3}{|c|}{ Destination sequence number } \\
\hline \multicolumn{3}{|c|}{ Originator IP address } \\
\hline \multicolumn{3}{|c|}{ Originator sequence number } \\
\hline \multicolumn{3}{|c|}{ Time stamp } \\
\hline
\end{tabular}

Fig. 1. RREQ message format

\begin{tabular}{|l|l|}
\hline Type & Reserved \\
\hline Broadcast ID \\
\hline Destination IP address \\
\hline Destination sequence number \\
\hline Originator IP address \\
\hline Time stamp \\
\hline
\end{tabular}

Fig. 2. R-RREQ message format

Table 1. Routing table

\begin{tabular}{lllll}
\hline Destination & $\begin{array}{l}\text { Source IP } \\
\text { address }\end{array}$ & $\begin{array}{l}\text { Destination } \\
\text { sequence number }\end{array}$ & $\begin{array}{l}\text { Hop } \\
\text { count }\end{array}$ & $\begin{array}{l}\text { Next hop } \\
\text { list }\end{array}$ \\
\hline
\end{tabular}

When the source node receives the RERR packet, it immediately removes the failed path in their routing table and it chooses another path on the routing table. If a single path between the source and destination is available then all the data packets are travel through the same path. Source node generates RREQ packet only for no path is available on the routing table. So it helpful to avoid extra overhead generated by a fresh route discovery and to reduce the route error transmission during route break recovery.

In randomized RAODV, source node selects the available path randomly in a routing table for data transfer to the destination node i.e., source node selects the different path in each time. In this study, compare the performance of AODV, RAODV and Randomized RAODV using the parameters like Packet Delivery Ratio, End to End Delay, Throughput, Packet Loss and Control Packet Overhead. The above metrics are helpful to analyze the performance of the Randomized RAODV. Packet delivery ratio value and Throughput value is high means the performance of the network is high; If the value of End to End delay, Control packet overhead and packet loss is low means degrade the better performance of the protocol.

\section{MATERIALS AND METHODS}

Simulations are helpful to evaluate the performance of the Randomized RAODV and also compare the performance of AODV, RAODV and Randomized RAODV. The simulation environment for performance analysis is shown in Table 2.

Detailed performance analysis of AODV, RAODV and Randomized RAODV using the parameters like Packet delivery ratio, Average end-to-end delay, Throughput, Packet loss, Control packet overhead. Two different scenarios are used to evaluate the above parameters. One scenario is varying the number of nodes but speed is constant and other is varying speed but node is constant.

Table 2. Simulation Parameters

\begin{tabular}{ll}
\hline Parameter & Value \\
\hline Simulator & ns- 2.34 \\
Protocols & AODV, RAODV, RRAODV \\
Number of nodes & $40,60,80,100,120,140$ \\
Simulation Area & $1000 \times 1000 \mathrm{~m}$ \\
MAC Layer & IEEE 802.11 \\
Radio Transmission range & $250 \mathrm{~m}$ \\
Movement Model & Random Way Point Model (RWP) \\
Traffic type & CBR \\
Mobility & $10 \mathrm{~ms}$ \\
Propagation & Two ray ground \\
Agent & UDP agent \\
Data Payload & 512 bytes/packet \\
\hline
\end{tabular}




\section{RESULTS}

\subsection{Scenario 1-Network with Varying Number of Nodes}

In scenario 1, AODV, RAODV and RRAODV are analyzed using the parameters like packet delivery ratio, end to end delay and Control packet overhead using varying the number of nodes $(40,60,80,100,120$ and $140)$ and the speed of the nodes is constant $\left(20 \mathrm{~m} \mathrm{~s}^{-1}\right)$.

\subsubsection{Packet Delivery Ratio (PDR)}

\section{Packet Delivery Ratio $=\sum$ Number of packet receive $/ \sum$ Number of packet send}

Figure 3 shows the packet delivery ratio and it is calculated as the ratio of packets delivered to the destination node to the total number of packets generated at the source node. When the number of nodes is minimum 40 nodes, PDR of Randomized RAODV has $96 \%$ and others have 52 and $68 \%$. When the number of nodes increased to 100 and 140, Packet delivery ratio of Randomized RAODV is decreased to 66 and $68 \%$. When the number of nodes increases, the mobility of nodes is also high. Randomized RAODV has better performance of the metric PDR for the number of nodes varies.

\subsubsection{End to End Delay}

End to End Delay $=\sum$ (arrive time-send time $) / \sum$ Number of connections

End to end delay means the ratio of inter arrival time between the two packets to the total packets delivery time. In Fig. 4, End to End delay is very low in Randomized AODV compared to AODV and RAODV. End to End delay of Randomized RAODV has around 97.33\% than AODV and $95.5 \%$ than RAODV.

\subsubsection{Control Packet Overhead}

In Fig. 5, Control packet overhead is less in Randomized RAODV compare to AODV and RAODV.

It measures the total number of control packets. Randomized RAODV has around $62 \%$ over in AODV and $35 \%$ over in RAODV.

\subsection{Scenario 2-Network with Varying Speed of Nodes}

In scenario 2, AODV, RAODV and RRAODV are analyzed using the parameters like packet delivery ratio, end to end delay and control packet overhead using varying the speed of nodes $\left(10,20,30,40\right.$ and $\left.50 \mathrm{~m} \mathrm{~s}^{-1}\right)$ with the number of nodes is constant (60).

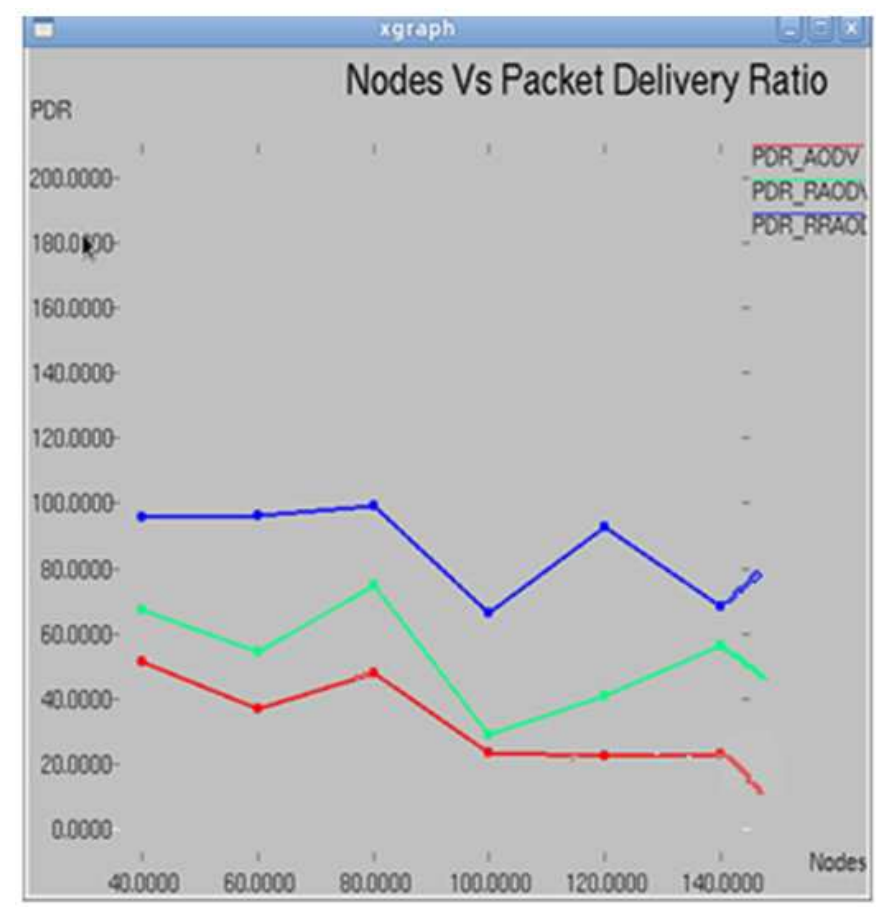

Fig. 3. Packet delivery ratio Vs number of nodes 
Santhi, K. and B. Parvathavarthini / Journal of Computer Science 10 (11): 1850-1858, 2014

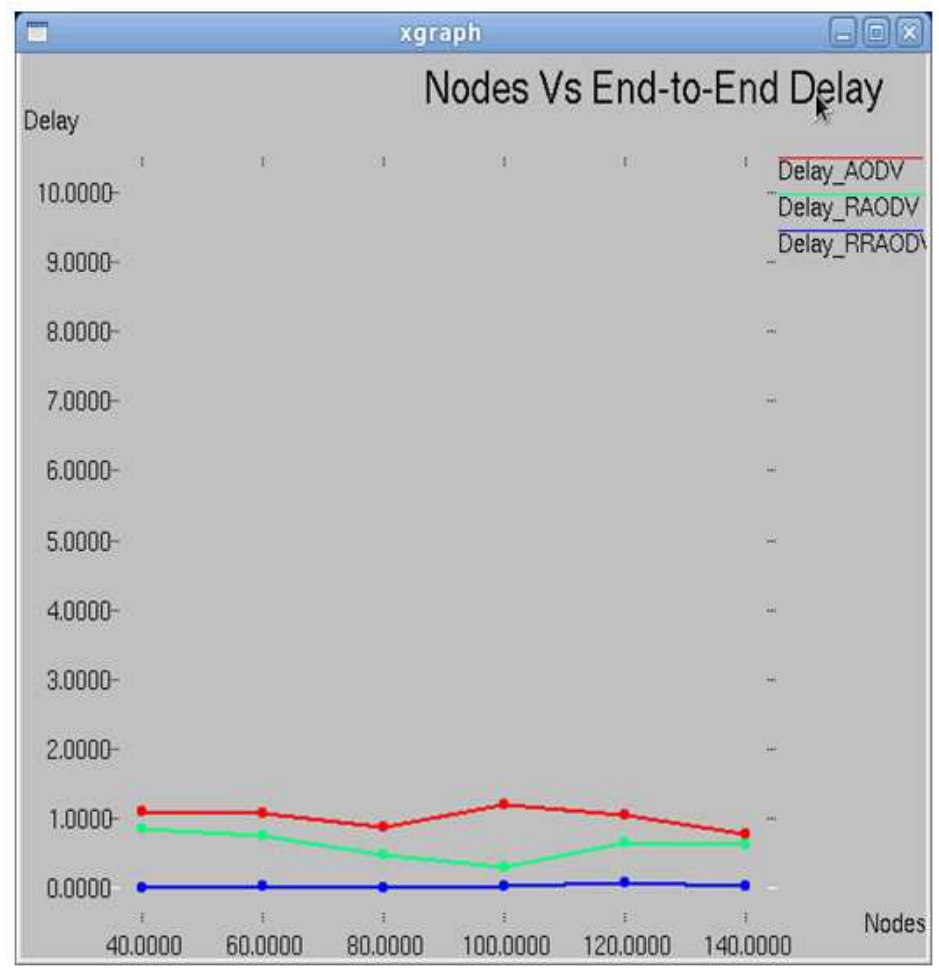

Fig. 4. End to end delay Vs number of nodes

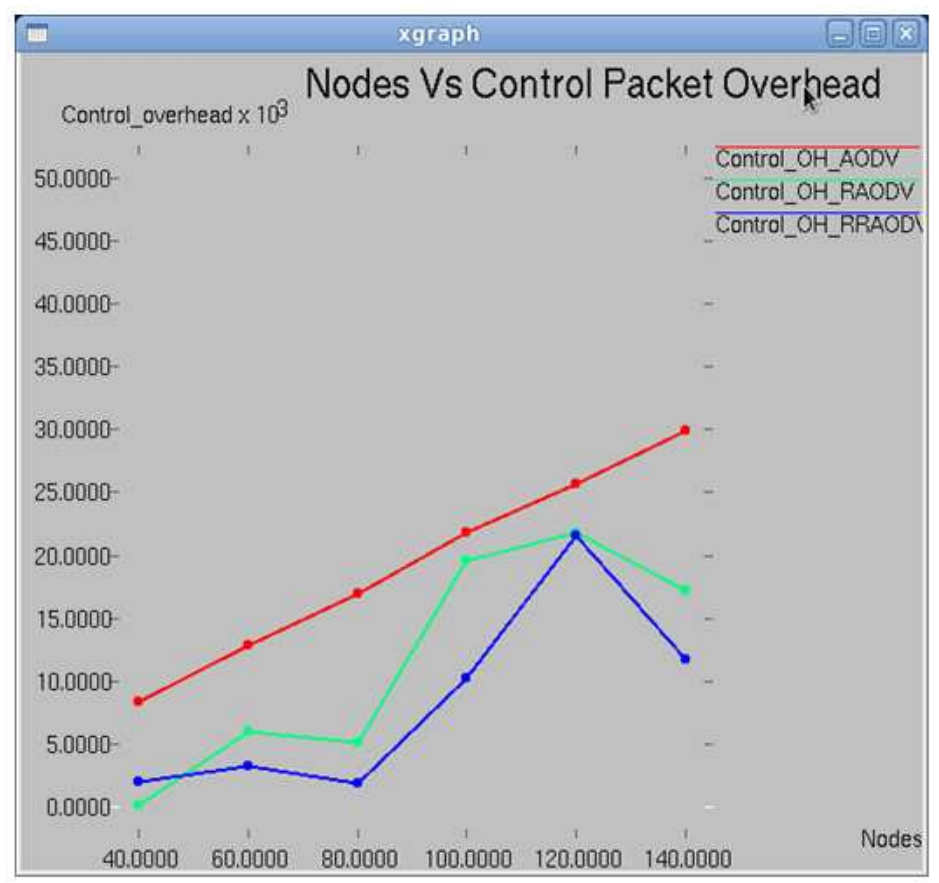

Fig. 5. Control packet overhead Vs number of nodes 
Santhi, K. and B. Parvathavarthini / Journal of Computer Science 10 (11): 1850-1858, 2014

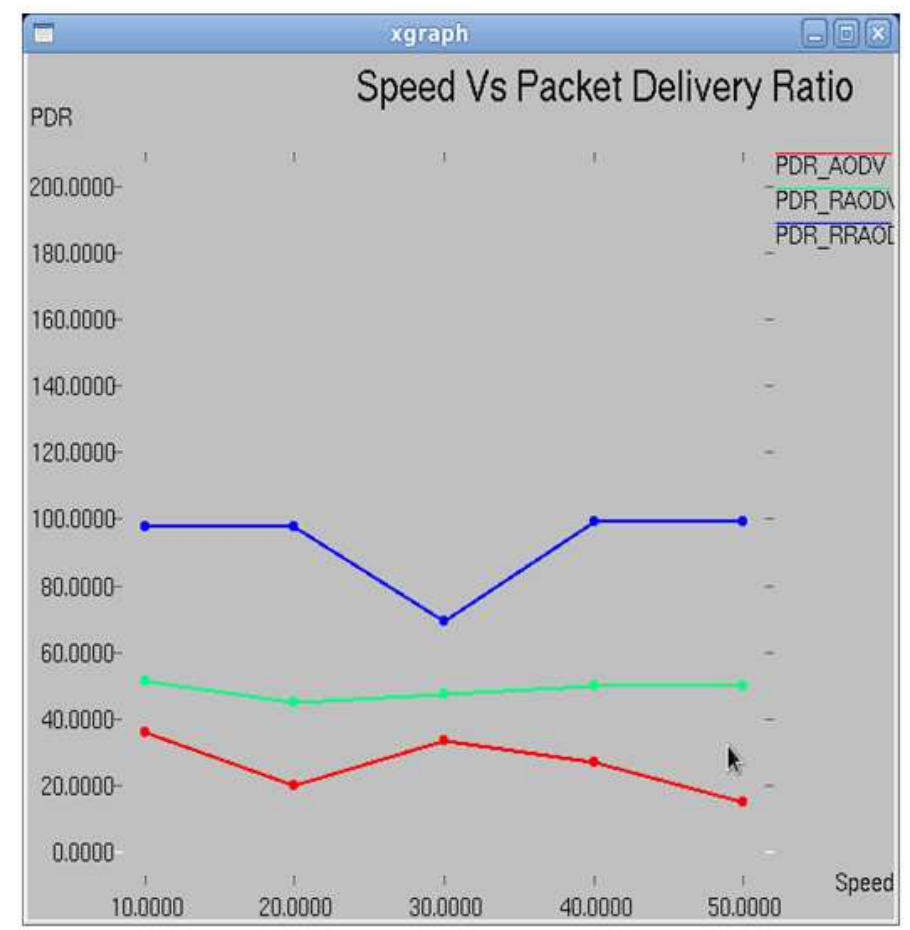

Fig. 6. Packet delivery ratio Vs speed

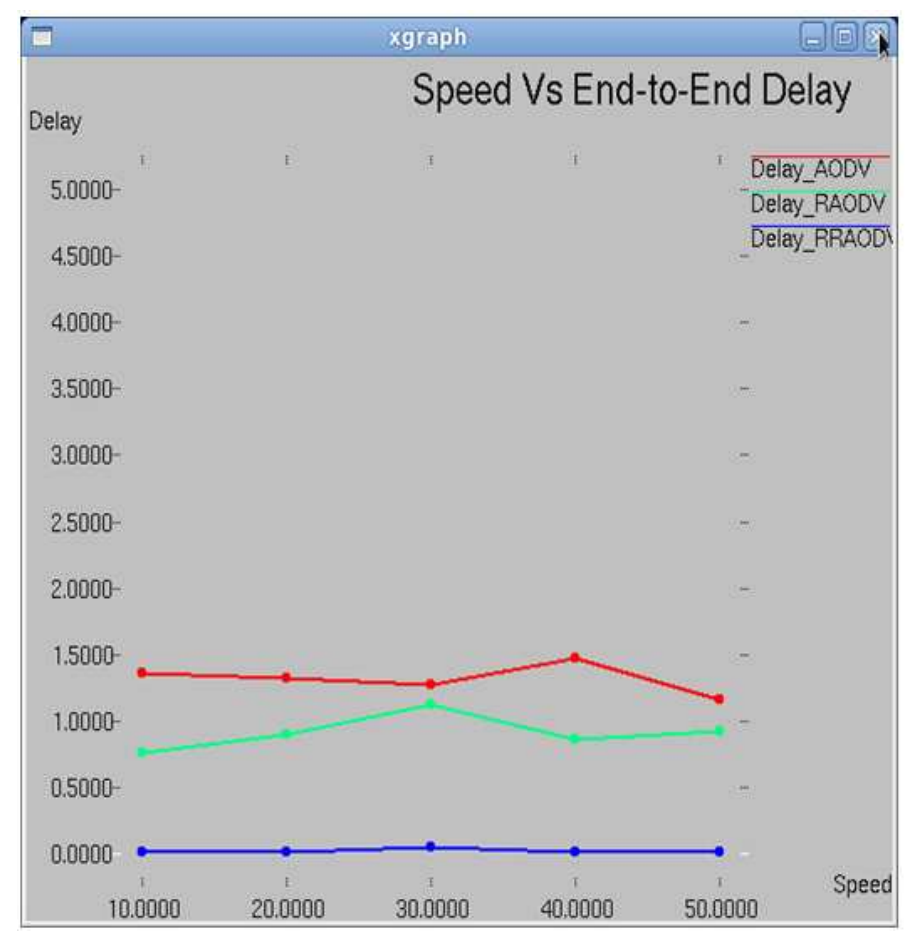

Fig. 7. End to end delay Vs speed 


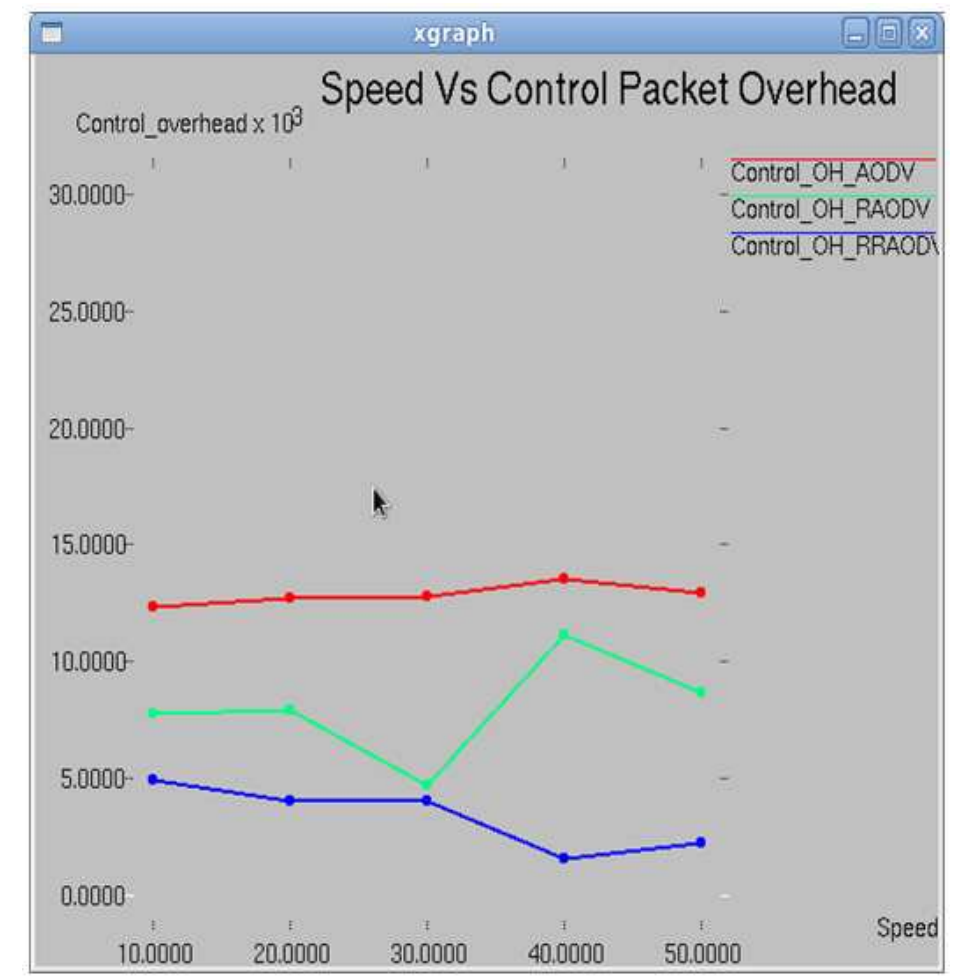

Fig. 8. Control packet overhead Vs speed

\subsubsection{Packet Delivery Ratio (PDR)}

Figure 6 shows the packet delivery ratio Vs Speed of AODV, RAODV and RRAODV. When the speed of the node is increased, packet delivery ratio is high in RRAODV compare to AODV and RAODV. RRAODV has $300 \%$ over AODV and $90.6 \%$ over RAODV.

\subsubsection{End to End Delay}

In Fig. 7, End to End delay is very low in Randomized AODV compared to AODV and RAODV.

End to End delay of AODV is nearly $1.31 \mathrm{~ms}$, RAODV has $0.91 \mathrm{~ms}$ but RRAODV has $0.03 \mathrm{~ms}$. End to End delay of Randomized RAODV has around 97.4\% than AODV and $97 \%$ than RAODV.

\subsubsection{Control Packet Overhead}

In Fig. 8, Control packet overhead is much reduced in Randomized RAODV compare to AODV and RAODV. Randomized RAODV has around $74 \%$ over in AODV and $52 \%$ over in RAODV.

\section{DISCUSSION}

Performance of the AODV, RAODV and Randomized RAODV is analyzed based on the number of nodes is represented in Table 3. Based on the result, Randomized RAODV has better performance on the nodes 40, 60 and 80 in the parameters packet delivery ratio, end to end delay and control packet overhead. When number of node is increased, the performance of the Randomized RAODV has changed.

In Table 3, using the parameter packet delivery ratio Randomized RAODV has $172.83 \%$ over AODV and $71.17 \%$ over RAODV. In End to end delay RRAODV has nearly $96 \%$ over AODV and RAODV. In control packet overhead, RRAODV has around 62\% over AODV and 35\% over RAODV.

Table 4 shows the analysis results of the performance of AODV, RAODV and Randomized RAODV based on the speed. Randomized RAODV has achieved high packet delivery ratio i.e., $300 \%$ over AODV and $90 \%$ over RAODV using various speed of the network and delay is 97\% over AODV and RAODV and also control packet overhead is $74 \%$ over AODV and $52 \%$ over RAODV. 
Table 3. Performance analysis of AODV, RAODV and randomized RAODV With respect to number of nodes

Speed $=20 \mathrm{~m} / \mathrm{s}$ (Constant)

Packet delivery ratio (\%) Vs No. of nodes

\begin{tabular}{|c|c|c|c|c|}
\hline $\begin{array}{l}\text { Number } \\
\text { of nodes }\end{array}$ & AODV & RAODV & $\begin{array}{l}\text { Randomized } \\
\text { RAODV }\end{array}$ & $\begin{array}{l}\text { Average efficiency of } \\
\text { randomized RAODV }\end{array}$ \\
\hline 40 & 52.000 & 68.000 & 96.000 & $172.83 \%$ over AODV \\
\hline 60 & 37.000 & 54.000 & 96.000 & $71.17 \%$ over RAODV \\
\hline 80 & 48.000 & 75.000 & 99.000 & \\
\hline 100 & 23.000 & 29.000 & 66.000 & \\
\hline 120 & 23.000 & 41.000 & 93.000 & \\
\hline 140 & 23.000 & 56.000 & 68.000 & \\
\hline \multicolumn{5}{|c|}{ End to end delay (ms) Vs no. of nodes } \\
\hline 40 & 1.096 & 0.847 & 0.006 & $97.33 \%$ over AODV \\
\hline 60 & 1.083 & 0.742 & 0.013 & $95.5 \%$ over RAODV \\
\hline 80 & 0.876 & 0.483 & 0.006 & \\
\hline 100 & 1.190 & 0.289 & 0.014 & \\
\hline 120 & 1.042 & 0.644 & 0.085 & \\
\hline 140 & 0.761 & 0.625 & 0.033 & \\
\hline \multicolumn{5}{|c|}{ Control packet overhead (Bytes) Vs no. of nodes } \\
\hline 40 & 8309.000 & 114.000 & 1998.000 & $61.83 \%$ over AODV \\
\hline 60 & 12891.000 & 6035.000 & 3293.000 & $34.5 \%$ over RAODV \\
\hline 80 & 16976.000 & 5145.000 & 1842.000 & \\
\hline 100 & 21879.000 & 19625.000 & 10183.000 & \\
\hline 120 & 25712.000 & 21815.000 & 21565.000 & \\
\hline 140 & 29898.000 & 17261.000 & 11771.000 & \\
\hline
\end{tabular}

Table 4. Performance analysis of AODV, RAODV and randomized RAODV With respect to speeds

Number of node $=60$ (Constant)

Packet delivery ratio $(\%)$ Vs speed $(\mathrm{m} / \mathrm{s})$

\begin{tabular}{|c|c|c|c|c|}
\hline Speed & AODV & RAODV & $\begin{array}{l}\text { Randomized } \\
\text { RAODV }\end{array}$ & $\begin{array}{l}\text { Average efficiency of } \\
\text { randomized RAODV }\end{array}$ \\
\hline 10 & 36.0000 & 51.000000 & 98.0000000 & $300 \%$ over AODV \\
\hline 20 & 20.00000 & 45.000000 & 98.0000000 & $90.6 \%$ over RAODV \\
\hline 30 & 33.00000 & 47.000000 & 69.0000000 & \\
\hline 40 & 27.00000 & 50.000000 & 99.0000000 & \\
\hline 50 & 15.00000 & 50.000000 & 99.0000000 & \\
\hline \multicolumn{5}{|c|}{ End to end delay $(\mathrm{ms}) \mathrm{Vs}$ speed $(\mathrm{m} / \mathrm{s})$} \\
\hline 10 & 1.35991 & 0.762293 & 0.0090940 & $97.4 \%$ over AODV \\
\hline 20 & 1.32318 & 0.892619 & 0.0102669 & $97 \%$ over RAODV \\
\hline 30 & 1.26603 & 1.126830 & 0.0528512 & \\
\hline 40 & 1.46916 & 0.855379 & 0.0087620 & \\
\hline 50 & 1.15514 & 0.916582 & 0.0664095 & \\
\hline \multicolumn{5}{|c|}{ Control packet overhead (Bytes) Vs speed (m/s) } \\
\hline 10 & 12360.00000 & 7743.000000 & 4955.0000000 & $97 \%$ over RAODV \\
\hline 20 & 12747.00000 & 7917.000000 & 4012.0000000 & $52 \%$ over RAODV \\
\hline 30 & 12775.00000 & 4714.000000 & 4001.0000000 & \\
\hline 40 & 13538.00000 & 11180.000000 & 1532.0000000 & \\
\hline 50 & 12925.00000 & 8648.000000 & 2229.0000000 & \\
\hline
\end{tabular}

\section{CONCLUSION}

In Randomized RAODV, the source node collects multiple paths from the destination node and all the path information is stored on the routing table based on hop count. The source node chose the path randomly in the routing table for the security purposes. Intruders cannot identify which way the data packets are travel to reach the destination node. The simulation work is based on the metrics for packet delivery ratio, throughput, control 
packet overhead, packet loss and end to end delay. Using these parameters, Randomized RAODV has better performance than AODV and RAODV with respect to the number of nodes and speeds. But bandwidth consumption is high in Randomized RAODV due to multiple path selection. Our future work will focus on introducing various attacks and then monitoring the performance of Randomized RAODV.

\section{REFERENCES}

Mtibaa, A. and F. Kamoun, 2006. MMDV: Multipath and MPR based AODV routing protocol. Proceedings of the IFIP 5th Annual Mediterranean Ad Hoc Networking Workshop, (HNW' 06), pp: 137-z144.

Gouda, B.S. and C.K. Behera, 2012. An enhanced approach for finding an optimal path in MANET using energy aware reverse reactive routing protocol (EA-RAODV). Int. J. Comput. Sci. Inform. Technolog., 3: 5280-5284.

Kuo, C.F., A.C. Pang and S.K. Chan, 2009. Dynamic routing with security considerations. IEEE Trans. Parall. Distrib. Syst., 20: 48-58. DOI: 10.1109/TPDS.2008.73

Kim, C., E. Talipov and B. Ahn, 2006. A reverse AODV routing protocol in ad hoc mobile networks. Proceedings of the International Conference on Emerging Directions in Embedded and Ubiquitous Computing, Aug. 1-4, Springer Berlin Heidelberg, Seoul, Korea, pp: 522-531. DOI: 10.1007/11807964_53

Talipov, E., D. Jin, J. Jung, I. Ha and C. Kim et al., 2006. Path hopping based on reverse AODV for security. Proceedings of the 9th Asia-Pacific International Conference on Network Operations and Management: Management of Convergence Networks and Services, Sept. 27-29, Springer Berlin Heidelberg, Busan, Korea, pp: 574-577. DOI: 10.1007/11876601_69

Jaisankar, N. and R. Saravanan, 2010. An extended AODV protocol for multipath routing in MANETs. Int. J. Eng. Technol., 2: 394-400.

Taleb, K. and K. Behzad, 2012. The effect of number of hops per path on remind energy in MANETs routing protocols. Int. J. Comput. Applic., 43: 23-28. DOI: 10.5120/6438-8848

Lee, S.J. and M. Gerla, 2001. Split multipath routing with maximally disjoint paths in ad hoc networks. Proceedings of the IEEE International Conference on Communications, Jun. 11-14, IEEE Xplore Press, Helsinki, pp: 3201-3205. DOI: 10.1109/ICC.2001.937262
Marina, M. and S. Das, 2001. On-demand multipath distance vector routing in ad hoc networks. Proceedings of the 9th International Conference on Network Protocols, Nov. 11-14, IEEE Xplore Press, pp: 14-23. DOI: 10.1109/ICNP.2001.992756

Zarei, M., K. Faez and J.M. Nya, 2008. Modified Reverse AODV routing algorithm using route stability in mobile ad hoc networks. Proceedings of the 12th IEEE International Multitopic Conference, Dec. 23-24, IEEE Xplore Press, Karachi, pp: 255259. DOI: 10.1109/INMIC.2008.4777745

Humaira, N., V. Krishna, S. Rao and S. Ahmed, 2011. Performance evaluation of on demand routing protocols AODV and modified AODV (R-AODV) in MANETS. Int. J. Distrib. Parallel Syst.

Kotzanikolaou, P., R. Mavropodi and C. Douligeris, 2005. Secure multipath routing for mobile ad hoc networks. Proceedings of the 2nd Annual Conference on Wireless On-Demand Network Systems and Services, Jan. 19-21, IEEE Xplore Press, pp: 89-96. DOI: 10.1109/WONS.2005.31

Sambasivam, P., A. Murthy and E.M. Belding-Royer, 2004. Dynamically adaptive multipath routing based on AODV. Proceedings of the 3rd Annual Mediterranean Ad hoc Networking Workshop Med Hoc Net, (MNH' 04) pp: 16-28.

Das, P., 2013. Comparison of AODV and R-AODV routing protocols by varying network mobility, network area and network density. Int. J. Emerg. Technol. Adv. Eng., 3: 472-477.

Khelifa, S. and Z.M. Maaza, 2010. An energy multi-path AODV routing protocol in ad hoc mobile networks. World Acad. Sci., Eng. Technol., pp: 1508-1512.

Vanitha, M. and B. Parvathavarthini, 2013. An enhanced DOA (DSR over AODV) protocol for mobile ad-hoc networks. Int. Rev. Comput. Software, 8: 14161416.

Li, X. and L. Cuthbert, 2004. On-demand node-disjoint multipath routing in wireless ad hoc networks. Proceedings of the 29th Annual IEEE International Conference on Local Computer Networks, Nov. 1618, IEEE Xplore Press, pp: 419-420. DOI: 10.1109/LCN.2004.90

Zangeneh, V. and S. Mohammadi, 2011. New multipath node-disjoint routing based on AODV protocol. World Acad. Sci. Eng. Technol. 